\title{
Le Quaternaire de la côte sud-est de la baie d'Ungava, Québec nordique
}

\author{
The Quaternary of the Southeastern Ungava Bay Coast,
} Northern Québec

\section{Das Quaternär der Südostküste der Ungava Bay, nordisches Québec}

\author{
Michel Allard, Alain Fournier, Emile Gahé et Maurice K. Seguin
}

Volume 43, numéro 3, 1989

Le $\mathrm{VI}^{\mathrm{e}}$ Congrès de l'Association québécoise pour l'étude du Quaternaire

URI : https://id.erudit.org/iderudit/032786ar

DOI : https://doi.org/10.7202/032786ar

\section{Aller au sommaire du numéro}

\section{Éditeur(s)}

Les Presses de l'Université de Montréal

\section{ISSN}

0705-7199 (imprimé)

1492-143X (numérique)

\section{Découvrir la revue}

\section{Citer cet article}

Allard, M., Fournier, A., Gahé, E. \& Seguin, M. K. (1989). Le Quaternaire de la côte sud-est de la baie d'Ungava, Québec nordique. Géographie physique et Quaternaire, 43(3), 325-336. https://doi.org/10.7202/032786ar

\section{Résumé de l'article}

La région étudiée s'étend de part et d'autre de l'estuaire du fleuve George et le long du littoral de la baie d'Ungava jusqu'au fjord Weymouth. On y a mesuré trois mouvements glaciaires: 1) les trains de broutures et des stries à un site situé sur une berge rocheuse du George témoignent d'un ancien mouvement glaciaire datant d'avant le Wisconsinien supérieur et orienté vers $280^{\circ}$, en provenance des monts Torngat; 2) les marques et les formes glaciaires dessinent un mouvement tournant, passant de 330e en amont de l'estuaire du George à 010e à son embouchure et 3) un dernier écoulement glaciaire, superposé au précédent, qui, décalé, passe de $350^{\circ}$ au sud à $030^{\circ}$ au nord. Le dernier écoulement glaciaire provenant du sud-est, soit des hauteurs le long de la frontière du Labrador, résulte d'un changement de dynamique glaciaire précédant de peu la déglaciation. Toutes les stries et les roches moutonnées observées le long de la côte est de la baie d'Ungava datent du dernier écoulement glaciaire en provenance des monts Torngat. De nombreux chenaux marginaux entaillés dans les tills des vallées lacustres du plateau témoignent d'une fonte glaciaire par attrition sur place. La limite de la Mer d'Iberville, à $100 \mathrm{~m}$ d'altitude, suit un plan horizontal d'amont en aval de l'estuaire, par suite de la déglaciation plutôt rapide du cours inférieur du George. Ce niveau marin maximal s'abaisse toutefois vers le nord-est, le long de la côte est de ia baie, probablement en raison de la présence de masses de glace moins importantes sur la péninsule du Labrador au Wisconsinien supérieur. La plus vieille datation au 14C, de $7380 \pm 90$ (UL-358), permet d'envisager, avec les données existantes sur la rive ouest de la baie, que la déglaciation a été à peu près simultanée tout le long des côtes de la baie d'Ungava. Quinze datations au $14 \mathrm{C}$ ont servi à bâtir une courbe de relèvement isostatique qui montre un tauxd'émersion de l'ordre de 4,5 m/siècle entre la déglaciation et $5800 \mathrm{BP}$, une inflexion à cette date et un taux très ralenti d'environ 0,5 m/siècle depuis, 


\section{LE QUATERNAIRE DE LA CÔTE SUD-EST DE LA BAIE D'UNGAVA, QUÉBEC NORDIQUE}

Michel ALLARD, Alain FOURNIER, Émile GAHÉ et Maurice K. SEGUIN; les trois premiers auteurs, Département de géographie et Centre d'études nordiques, et le quatrième auteur, Département de géologie et Centre d'études nordiques, Université Laval, Sainte-Foy, Québec G1K 7P4.

RÉSUMÉ La région étudiée s'étend de part et d'autre de l'estuaire du fleuve George et le long du littoral de la baie d'Ungava jusqu'au fjord Weymouth. On y a mesuré trois mouvements glaciaires: 1) les trains de broutures et des stries à un site situé sur une berge rocheuse du George témoignent d'un ancien mouvement glaciaire datant d'avant le Wisconsinien supérieur et orienté vers $280^{\circ}$, en provenance des monts Torngat; 2) les marques et les formes glaciaires dessinent un mouvement tournant, passant de $330^{\circ}$ en amont de l'estuaire du George à $010^{\circ}$ à son embouchure et 3 ) un dernier écoulement glaciaire, superposé au précédent, qui, décalé, passe de $350^{\circ}$ au sud à $030^{\circ}$ au nord. Le dernier écoulement glaciaire provenant du sud-est, soit des hauteurs le long de la frontière du Labrador, résulte d'un changement de dynamique glaciaire précédant de peu la déglaciation. Toutes les stries et les roches moutonnées observées le long de la côte est de la baie d'Ungava datent du dernier écoulement glaciaire en provenance des monts Torngat. De nombreux chenaux marginaux entaillés dans les tills des vallées lacustres du plateau témoignent d'une fonte glaciaire par attrition sur place. La limite de la Mer d'íberville, à $100 \mathrm{~m}$ d'altitude, suit un plan horizontal d'amont en aval de l'estuaire, par suite de la déglaciation plutôt rapide du cours inférieur du George. Ce niveau marin maximal s'abaisse toutefois vers le nord-est, le long de la côte est de la baie, probablement en raison de la présence de masses de glace moins importantes sur la péninsule du Labrador au Wisconsinien supérieur. La plus vieille datation au ${ }^{14} \mathrm{C}$, de $7380 \pm 90$ (UL358), permet d'envisager, avec les données existantes sur la rive ouest de la baie, que la déglaciation a été à peu près simultanée tout le long des côtes de la baie d'Ungava. Quinze datations au ${ }^{14} \mathrm{C}$ ont servi à bâtir une courbe de relèvement isostatique qui montre un taux d'émersion de l'ordre de $4,5 \mathrm{~m} /$ siècle entre la déglaciation et $5800 \mathrm{BP}$, une inflexion à cette date et un taux très ralenti d'environ $0,5 \mathrm{~m} /$ siècle depuis.
ABSTRACT The Quaternary of the southeastern Ungava Bay coast, northern Québec. The study area extends on both sides of the George River Estuary and along the Ungava Bay coast as far as Weymouth Inlet. Three ice flow directions have been measured: 1) striations, crescentic gouges and crescentic fractures on one site on the rocky shore of George River indicate an ancient ice flow (probably older than the Late Wisconsinan) direction toward $280^{\circ}$, most probably originating from the Torngat Mountains: 2) striations, grooves, fluted tills and drumlins draw a broad curve, imaging a flow towards $330^{\circ}$ in the upstream region that rotated to $010^{\circ}$ at the river mouth; and 3) a last flow pattern is superimposed over the preceding one with a $20^{\circ}$ angular shift, thus turning from $350^{\circ}$ in the south to $030^{\circ}$ in the north. The very last ice flow originated from the southeast, i.e. from the highlands along the LabradorQuébec border. All striations and roches moutonnées surveyed along the eastern coast of Ungava Bay were made by the last ice flow originating from the Torngat Mountains. Numerous ice marginal channels in till within lake basins suggest in situ decay of the ice sheet. The $100 \mathrm{~m}$ high limit of d'lberville Sea has a horizontal plane along the George River Estuary, suggesting a rather rapid deglaciation. This highest shoreline is downtilted towards the northeast probably due to a limited ice load over the Labrador Peninsula during the Late Wisconsinan. The oldest ${ }^{14} \mathrm{C}$ date is $7380 \pm 90 \mathrm{BP}$ (UL-358), from marine shells at the very base of the marine clays. It suggests, with other dates in the literature that all Ungava Bay was deglaciated simultaneously. Fifteen ${ }^{14} \mathrm{C}$ dates are used to build a sea level curve which indicates a fast emergence rate of about $4.5 \mathrm{~m} /$ century from deglaciation to $5800 \mathrm{BP}$, an inflexion at that time and a much lower rate $(0.5 \mathrm{~m} /$ century) since then.
ZUSAMMENFASSUNG Das Quaternär der Südostküste der Ungava Bay, nordisches Québec. Das untersuchte Gebiet erstreckt sich beidseitig der Gezeitenmündung des George-Flusses und das ganze Küstengebiet der Ungava-Bay entlang bis zum Fjord Weymouth. Man hat dort drei glaziale Bewegungen gemessen: 1) der Veriauf der Schübe und Schrammen eines auf einem Felsufer des George-Flusses gelegenen Platzes bezeugt eine alte glaziale Bewegung von vor dem späten Wisconsinium, die in Richtung $280^{\circ}$ von den Torngat-Bergen ausging; 2) die glazialen Spuren und Formen zeichnen eine Drehbewegung ab, die von $330^{\circ}$ oberhalb der Gezeitenmündung des George zu $010^{\circ}$ an seiner Mündung wechselte und 3) ein letztes glaziales Fliessen welches das vorige versetzt überlagert und sich von $350^{\circ}$ im Süden bis $030^{\circ}$ im Norden dreht. Das letzte, vom Südosten ausgehende glaziale Fliessen, also von den Höhen entlang der Grenze von Labrador, wurde durch einen Wechsel der glazialen Dynamik kurz vor der Enteisung hervorgerufen. Alle entlang der Ostküste der Ungava-Bay beobachteten Schrammen und Schäfchenfelsen stammen aus der Zeit des letzten glazialen Fliessens von den TorngatBergen. Zahlreiche Randkanäle, welche in die Tills der Seentäler des Plateaus eingeschnitten sind, bezeugen ein glaziales Schmelzen durch Zerreibung vor Ort. Die Grenze des Meers von lberville in $100 \mathrm{~m}$ Höhe folgt einer Horizontalebene oberhalb und unterhalb der Gezeitenmündung infolge der eher schnellen Enteisung des unterlaufs des George. Dieses maximale marine Niveau senkt sich indessen nach Nordosten entlang der Ostküste der Bay. Die älteste ${ }^{14} \mathrm{C}$-Datierung von $7380 \pm 90$ (UL-358) führt zusammen mit den vorhandenen Daten am Westufer der Bay zu der Annahme, dass die Enteisung entlang der Küste der Ungava-Bay etwa gleichzeitig stattgefunden hat. Mit fünfzehn ${ }^{14} \mathrm{C}$-Datierungen wurde eine Kurve der isostatischen Hebung erstellt, die einen Auftauchgrad der Grösse 4,5m/Jh. zeigt zwischen der Enteisung und 5800 v.u.Z., eine Biegung zu diesem Zeitpunkt und seitdem eine Verlangsamung von ungefähr $0,5 \mathrm{~m} / \mathrm{Jh}$. 


\section{INTRODUCTION}

L'obtention de nouvelles données sur le Quaternaire des territoires bordant les façades sud et est de la baie d'Ungava s'avère essentielle à une meilleure connaissance d'une portion très importante de l'Inlandsis laurentidien. En effet, cette région se situe, d'une part, à proximité des monts Torngat et de la côte du Labrador où l'inlandsis a connu sa limite maximale au Wisconsinien supérieur (Ives, 1978; Ives et al., 1976; Dyke et Prest, 1987) et d'autre part, à quelques centaines de kilomètres à peine du centre de la péninsule du Québec-Labrador où eut lieu la fonte finale du glacier (Richard et al., 1982). De plus, l'âge de la déglaciation des environs de l'estuaire du fleuve George revêt une importance capitale pour la compréhension de l'évolution des grands lacs glaciaires du nordest du Québec-Labrador, en particulier le Lac Naskaupi qui occupa le cours supérieur du George durant la déglaciation (Ives, 1960a; Barnett, 1963, 1967). En effet, l'établissement de l'âge minimal de la déglaciation permet de mieux comprendre à la fois l'histoire de ces lacs glaciaires et les étapes de déglaciation de la baie d'Ungava elle-même.

La région étudiée s'étend de part et d'autre de l'estuaire du fleuve George, qui fait environ 65 kilomètres de longueur (fig. 1). Des observations, principalement des mesures d'orientation de stries glaciaires et des mesures de l'altitude de la limite marine postglaciaire, ont aussi été faites au cours de reconnaissances en embarcation le long des côtes rocheuses et sur de nombreux îlots de la baie d'Ungava, particulièrement autour de l'embouchure du Koroc et dans la région des fjords Davis, Gregson et Weymouth (fìg. 1). Aucun relevé de la géologie du Quaternaire n'avait été effectué dans les environs depuis les travaux de Matthew (1961a et b) dans la région des collines Pyramides, à $60 \mathrm{~km}$ en amont de la région étudiée.

La présente étude fait d'abord état des indicateurs d'écoulements glaciaires mesurés le long de l'estuaire du George et de la côte de la baie d'Ungava pour ensuite aborder le problème de la déglaciation et de la transgression de la Mer d'lberville. On discute aussi des implications des nouvelles observations sur les concepts de la déglaciation du nord-est du Québec-Labrador.

\section{GÉOLOGIE DU PRÉCAMBRIEN ET MORPHOLOGIE PRÉ-GLACIAIRE}

La région étudiée appartient à l'arrière-pays de la province structurale de Churchill. Les roches datent de l'Archéen et ont été métamorphisées lors de l'orogenèse hudsonienne (Taylor, 1971, 1974). Au moins deux phases de plissements sont responsables de la complexité structurale des gneiss de la région. Aux abords de l'estuaire du George, le socle rocheux est constitué principalement de gneiss granitique et granodioritique. On trouve aussi des amphibolites, des schistes à biotites et quelques filons de granite, de diabase et de gabbro. Ce contexte géologique, répétitif sur l'ensemble de la région, favorise peu l'utilisation de lithologies particulières comme traceurs dans les dépôts glaciaires.

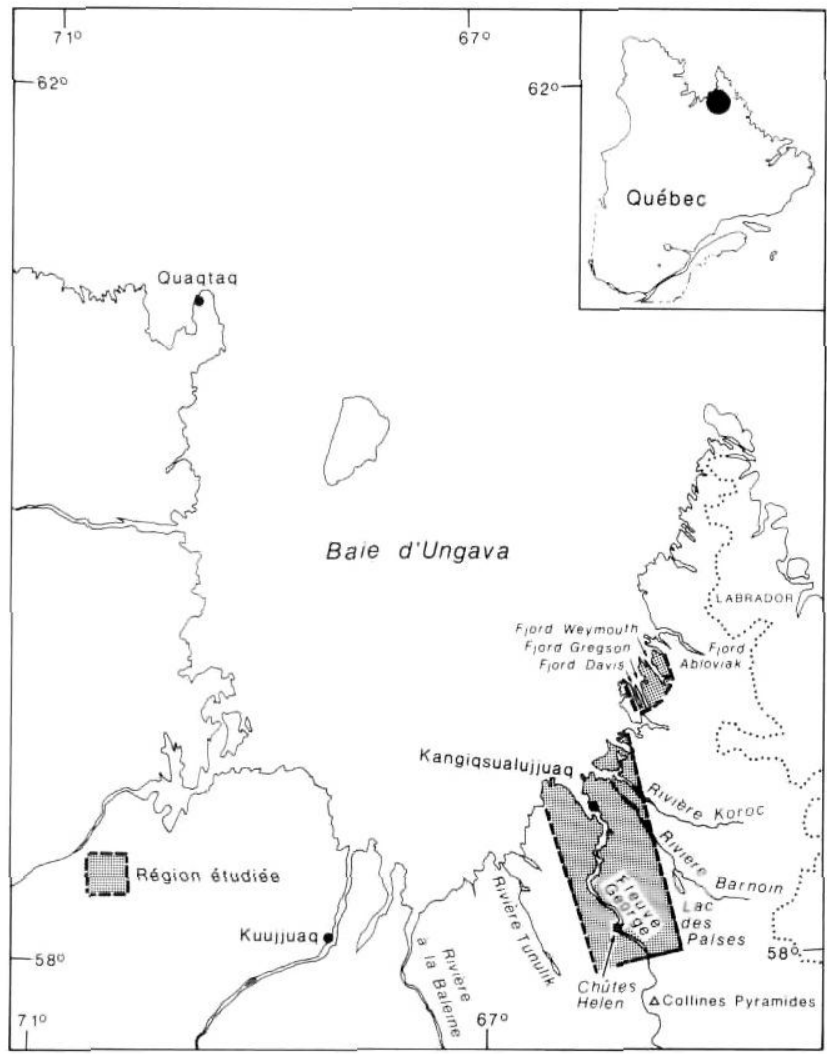

FIGURE 1. Localisation de la région étudiée et noms de lieux. Certains toponymes à l'intérieur de la région étudiée apparaissent à la figure 2.

Location of the study area and place names. Some of the names within the study area are on Figure 2.

Le plateau du George (Québec, 1983) est une surface de pénéplaine ancienne et basculée qui s'incline depuis les monts Torngat du Labrador jusqu'à la plaine de la rivière à la Baleine, qui s'étend entre la rivière Tunulic et Kuujjuaq. Le fleuve George occupe un sillon profond qui entaille ce plateau du sud au nord interceptant ainsi le drainage des terres en provenance des monts Torngat. La vallée est encaissée entre des parois hautes de quelque $100-200 \mathrm{~m}$. La surface du plateau, bosselée, est à une altitude moyenne de 300-400 m, tandis que le relief est morcelé en vallées et en collines allongées le long de l'estuaire à partir de Kangiqsualujjuaq. Ces reliefs oblongs sont orientés les uns à environ $310^{\circ}$, les autres à environ $332^{\circ}$. Selon les observations sommaires réalisées par Fournier (1988), en l'absence d'une carte géologique détaillée, ces orientations du relief correspondraient aux deux directions axiales de plissements régionaux. Ces reliefs rocheux sont grossièrement parallèles aux formes profilées et peuvent avoir localement canalisé l'écoulement de la glace lorsqu'elle s'est amincie avant la déglaciation finale.

Le long de la côte est de la baie d'Ungava, le plateau rocheux est perché au-dessus d'un littoral à skjars et entaillé de fjords qui, du sud vers le nord jusqu'au fjord Abloviak, deviennent de plus en plus encaissés. 


\section{LES DIRECTIONS D'ÉCOULEMENT GLACIAIRE}

Cartographiés d'après des photographies aériennes (fig. 2), les drumlins et les tills cannelés, abondants sur le plateau de part et d'autre du George, fournissent une image d'ensemble des dernières directions d'écoulement glaciaire. Toutefois, seules quelques rares formes orientées ont été repérées sur les photos aériennes dans le secteur de la péninsule labra-

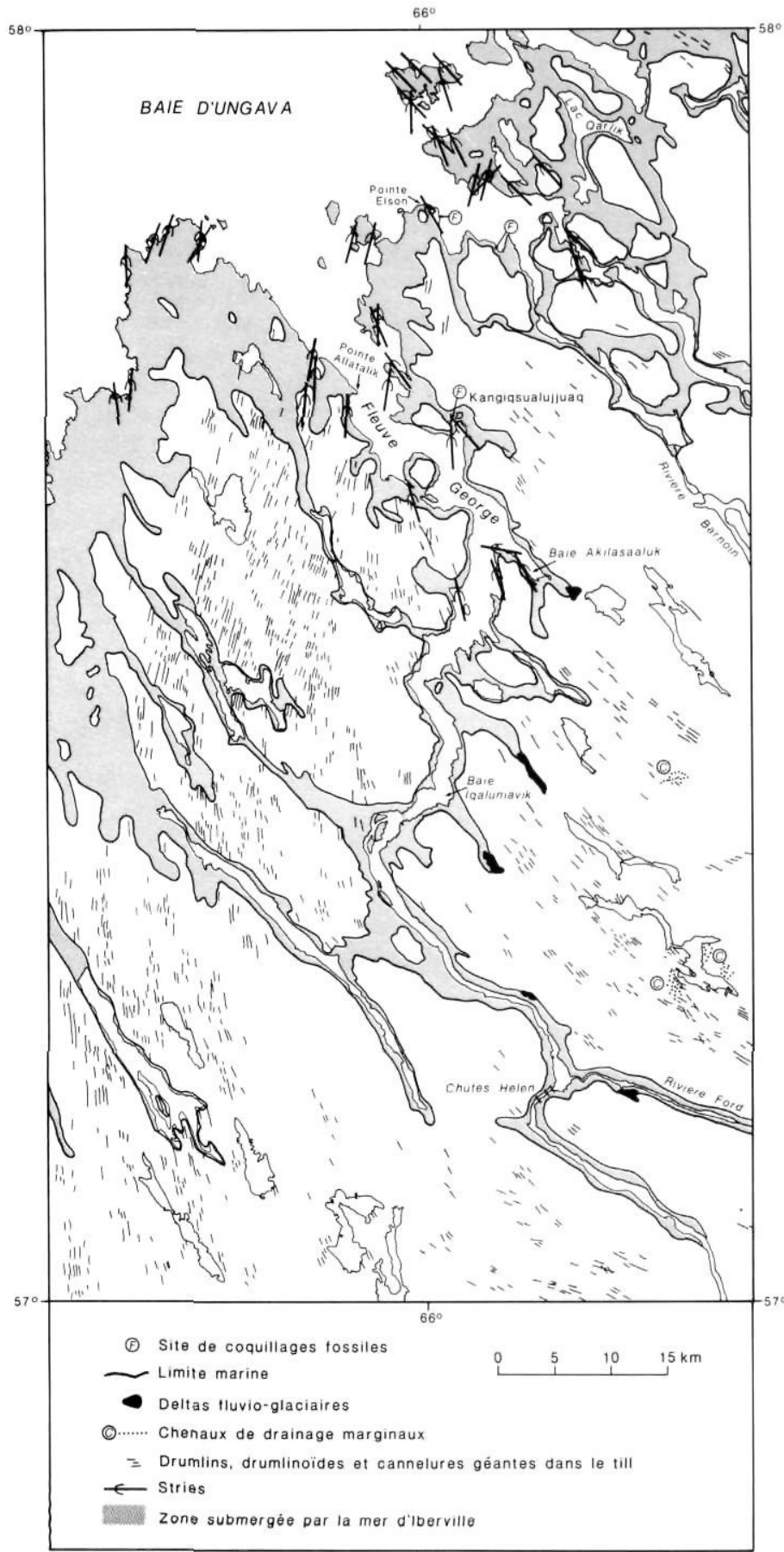

FIGURE 2. Écoulements glaciaires et limite de la Mer d'Iberville de part et d'autre du cours inférieur du fleuve George.

Glacial flow indicators and limit of postglacial d'Iberville Sea in the vicinity of the lower reaches of George River. doréenne s'étendant jusqu'au fjord Weymouth parce que le terrain y est principalement rocheux. Le relevé des stries, des trains de broutures et des cannelures au niveau des affleurements rocheux permet des observations plus précises, en particulier lorsque deux directions se recoupent ou que les affleurements ont deux faces polies (Veillette, 1986). Les surfaces rocheuses exposées à l'air depuis longtemps étant en général très altérées, c'est dans la zone intertidale de l'estuaire et des îles que la plupart des mesures ont été prises.

Un affleurement rocheux situé sur une pointe à l'entrée sud de la baie d'Akilasaaluk, à 12 km en amont de Kangiqsualujjuaq, porte des stries et des broutures qui indiquent un écoulement glaciaire orienté vers $280^{\circ}$ (moyenne de plusieurs mesures entre $265^{\circ}$ et $285^{\circ}$ ) (fig. 3). L'affleurement s'étend au pied d'une falaise en dépôts meubles de $8,5 \mathrm{~m}$ de hauteur sous lesquels la surface polie se poursuit. Revisité en 1988, l'affleurement est présentement en voie de démantèlement rapide par suite de l'action des processus d'érosion littorale périglaciaire (Fournier, 1988). La coupe de la falaise est unique par sa stratigraphie; on y trouve de bas en haut: (a) 1,5 m de sable graveleux stratifié et oxydé à cailloux subanguleux et sub-arrondis; les strates de quelques décimètres d'épaisseur sont plissées, apparemment par la glacitectonique, (b) une couche de till, lui aussi plissé, de $50 \mathrm{~cm}$ d'épaisseur, (c) 2,2 m de petits graviers stratifiés, plissés eux aussi, (d) $30 \mathrm{~cm}$ de sable grossier bien trié et oxydé et (e) $4 \mathrm{~m}$ de till blocailleux à matrice sableuse, typique du faciès régional du till du Wisconsinien supérieur. Les unités $a, b$, c et $d$ de la coupe peuvent être réunies en une seule entité puisqu'elles partagent, malgré les variations sédimentologiques, les mêmes déformations glacitectoniques. Elles appartiennent vraisemblablement à un dépôt fluvial ou fluvio-glaciaire antérieur au till régional, déformé par la glacitectonique auquel s'est incorporée une couche de till lors de l'avancée glaciaire.

Comme l'affleurement strié se trouve en dessous de ces dépôts, il est possible que le mouvement glaciaire qu'on y

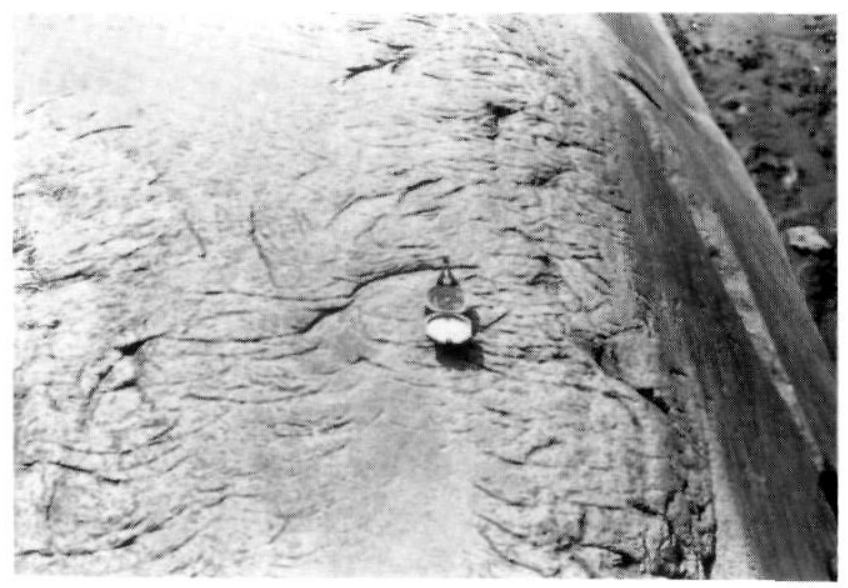

FIGURE 3. Broutures, marques en croissant et stries orientées vers $280^{\circ}$ près de l'entrée de la baie d'Akilasaaluk.

Gouges, crescentic marks and striations oriented towards $280^{\circ}$ near the entrance of Akilasaaluk Bay. 
détecte soit plus ancien que la dernière avancée glaciaire régionale. Non daté, ce mouvement glaciaire ancien est venu de l'est, depuis les hauts plateaux et les monts Torngat. II pourrait correspondre à la dernière englaciation ou être plus vieux.

L'ensemble des drumlins, des tills cannelés et des polis glaciaires dessine un arc de cercle, déjà esquissé sur la carte glaciaire du Canada (Prest et al., 1968). Le relevé détaillé de ces formes orientées révèle toutefois que deux mouvements glaciaires se sont succédé dans la région de l'estuaire du George. Le premier de ces mouvements orienté à $330^{\circ}$ en amont du fleuve, de part et d'autre des chutes Helen, dessine un arc de cercle, et passe à $000^{\circ}$ près de la pointe d'Allatalik puis à $010^{\circ}$ à l'embouchure, voire à $014^{\circ}$ sur les îles un peu au large. Le second mouvement glaciaire décrit une courbe similaire mais selon des orientations décalées vers la droite d'une vingtaine de degrés, passant de $350^{\circ}$ au sud à $030^{\circ}$ au nord. Une direction de $045^{\circ}$, associée à ce dernier écoulement, a même été mesurée sur le rivage d'une péninsule dans la baie d'Ungava (fig. 2).

L'écoulement vers le nord dans les deux cas ne fait aucun doute, compte tenu des nombreux trains de broutures et des abondantes fractures en croissant relevées sur les affleurements polis. Cette direction est aussi évidente dans les nombreux crag-and-tails géants associés aux champs de drumlins et dont les traînées de till sculptées à l'abri de buttes rocheuses s'étirent vers le nord.

L'âge relatif des deux mouvements glaciaires est facile à déterminer par l'analyse en trois sites de drumlins superposés qui montrent clairement le refaçonnement de drumlins anciens par un remodelage superficiel du till suivant une nouvelle direction (fig. 4). Cette datation relative est réalisable, plus difficilement cependant au niveau des stries et des affleurements à deux faces tronquées; elle se complique du fait que les directions changent d'un bout à l'autre de la région et ce n'est qu'après la compilation à l'échelle régionale qu'une interprétation fiable peut être avancée. Le dernier mouvement glaciaire est celui qui a laissé les plus vastes champs de drumlins, de tills cannelés ainsi que quelques moraines de Rogen cannelées.

Ces deux mouvements glaciaires provenaient des hauts plateaux du George sis au sud-est de la région étudiée. Selon les cartes paléogéographiques de Dyke et Prest (1987), ils auraient émané de la crête de dispersion appelée "Ancestral Labrador ". Aucun autre mouvement plus récent ou orienté de façon différente pouvant être associé à une avancée glaciaire sur la baie d'Ungava, scellant ainsi le cours inférieur du George, n'a été observé sur le terrain. II appert donc que les mouvements giratoires observés, dirigés vers le NO et tournant vers le NE dans le cours inférieur du George, soient les derniers à avoir touché la région.

Bien qu'une étude détaillée du till régional dans les coupes le long du fleuve reste à faire, celui-ci ne montre pas de discordance ou de discontinuités stratigraphiques évidentes que l'on pourrait associer aux deux mouvements glaciaires superposés. II n'y a donc très probablement pas eu de recul glaciaire entre les deux phases d'écoulement, mais plutôt un

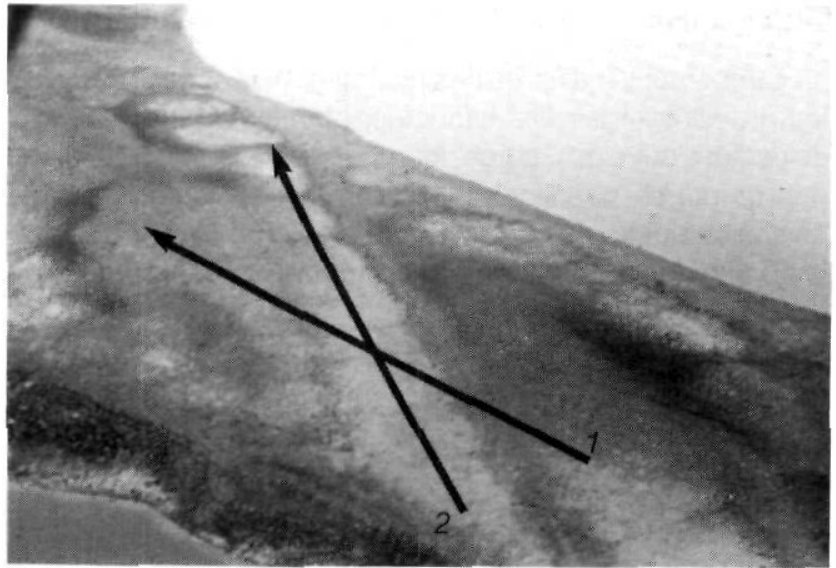

FIGURE 4. Superposition de deux directions d'écoulement glaciaire dans le till drumlinoïsé: 1) la plus ancienne, 2) la plus récente.

Crossing drumlin ridges: 1) oldest direction, 2) latest direction.

changement dans la distribution des masses de glace autour de la région, changement qui a dû provoquer assez rapidement une réorientation de la dynamique glaciaire.

Les reconnaissances effectuées sur la côte est de la baie d'Ungava n'ont permis l'observation que de directions d'écoulement vers le nord-ouest, soit des monts Torngat vers la baie. Ces quelques observations concordent avec celles de Ives rapportées par Loken (1964, p. 7 et 8).

\section{GÉOMORPHOLOGIE ET FACIĖS DES DÉPÔTS QUATERNAIRES}

\section{LES DÉPÔTS GLACIAIRES}

II n'existe de première évidence qu'un seul till dans la région selon les coupes étudiées. Cependant, aucune analyse d'orientations de cailloux n'a été faite pour vérifier si les directions d'écoulement superposées enregistrées par les formes sont détectables dans les coupes de till. Ces dépôts ont une matrice sableuse et contiennent de nombreux blocs striés. Les plus belles coupes sont dans les falaises le long du fleuve George. Une matrice sablo-limoneuse caractérise les 10 mètres supérieurs des coupes, tandis que le faciès devient graduellement plus sableux vers le bas tout en contenant des strates de sable discontinues et d'épaisseur variable de 2 à $20 \mathrm{~cm}$; certaines de ces couches de sable s'insinuent autour des blocs striés ou entre eux. Ces faciès tirent leur origine d'une mise en place dans un milieu sous-glaciaire comportant des écoulements lamellaires d'eau diffus et sporadiques. Les dépôts glaciaires reposent sur le roc qui peut être observé à la base de plusieurs coupes.

Lorsqu'il n'est pas découpé par les terrasses fluviales ou marines, le till prend, sur le plateau, la forme de drumlins, de moraines de Rogen et de placages épais. Sur les versants escarpés, il est disposé en longues bandes qu'on pourrait au premier coup d'œil confondre avec des langues de gélifluxion. L'observation de mouvements de versants actifs démontre qu'il s'agit plutôt d'une couverture épaisse de till sur les versants qui a été entaillée par de nombreux couloirs d'érosion parallèles. 
Le till épais, en particulier celui contenu dans les drumlins, est entaillé en trois endroits (fig. 2) de chenaux parallèles à des altitudes décroissantes dans les cuvettes profondes du plateau du George. Ces vallons aux réseaux non hiérarchisés ont été creusés par les eaux de fusion s'écoulant en marge de masses glaciaires résiduelles. Tout comme plus au sud dans la région de Schefferville (Henderson, 1959) et du lac Helluva (Ives, 1960b), ils témoignent d'une fonte sur place du glacier par attrition, le morcellement final des masses de glace étant commandé par la topographie locale.

Quelques très longs eskers s'allongent parallèlement aux drumlins. Sinueux et parfois anastomosés aux altitudes supérieures à la limite marine, ils se terminent ou s'élargissent en deltas lorsqu'ils franchissent cette limite, à l'endroit de leur ancienne embouchure dans la Mer d'lberville. Ces dépôts consistent en graviers très grossiers, stratifiés et sub-anguleux.

\section{LES FORMES ET LES DÉPÔTS DE LA MER D'IBERVILLE}

Les dépôts d'eau profonde ou calme sont peu abondants dans la région. Outre dans quelques coupes dans des falaises marines où on les aperçoit sous des sédiments grossiers, ils se retrouvent surtout le long de l'estuaire moyen du George dans le fond de vallées ou de cuvettes profondes qui, formant autrefois des baies aux ouvertures étroites, ont pu constituer des bassins favorables à la décantation de particules fines. Les limons marins sont ainsi présents au fond des baies de Kangiqsualujjuaq, d'Akilasaaluk et d'Iqaluniavik (fig. 2) de même que sous quelques tourbières à palses (Gahé et al., 1987). Forés à cinq endroits suivant la méthode hydraulique (Veillette et Nixon, 1980), ces limons argileux atteignent des épaisseurs de 14 à $30 \mathrm{~m}$.

Les faciès littoraux ou intertidaux sont très variés, hétérométriques et changeants latéralement. La plupart sont blocailleux. Le très grand marnage marégraphique, actuellement de $13 \mathrm{~m}$ aux vives-eaux, et les glaces flottantes ont créé un ensemble de conditions hydrodynamiques qui, tout au long de l'émersion holocène, furent responsables de courants de marée rapides agissant dans de vastes zones intertidales sur lesquelles de nombreux blocs et semis glaciels étaient charriés et déposés chaque année. On trouve dans les anciennes baies aujourd'hui soulevées de vastes zones encore sillonnées de chenaux de marée fossiles (fig. 5) et étalées sur des diamictons à matrice mal triée de limon et de sable très blocailleux. L'expansion du pergélisol dans ces dépôts se manifeste principalement par le développement de vastes champs de thufurs.

D'anciennes boulder barricades ou crêtes de blocs glaciels marquent ici et là dans la région des paléolignes de rivage prédominantes. Actuellement, ces formes s'observent dans la partie supérieure de zones intertidales à l'intérieur de baies ouvertes vers le large. Les plus spectaculaires, comme celle de la vallée de Kangiqsualujjuaq, constituent des seuils topographiques dans des vallées d'où la mer s'est graduellement retirée par les deux extrémités; il s'agit en quelque sorte de flèches ou de paléo-tombolos glaciels (fig. 6).

Les plages de galets émoussés et triés sont rares quoique présentes dans d'anciennes baies ouvertes vers la haute mer. Quant aux successions de plages sableuses et gra- veleuses, elles sont particulièrement abondantes autour des reliefs rocheux près de l'embouchure du George. Non fossilifères, elles sont constituées de plusieurs mètres de sable très grossier et de petit gravier stratifié de façon diffuse.

\section{LES SÉDIMENTS FLUVIATILES}

Peu abondants dans la région, les sédiments fluviatiles s'étalent sur le replat des terrasses étagées le long du George. Constituées de sables bien triés, ils reposent en discordance très nette sur des surfaces d'érosion taillées dans le till; un dallage de pierres se situe souvent à leur base. Ils ne font que de 1 à 1,5 m d'épaisseur.

\section{LES DÉPÔTS TOURBEUX ET RÉCENTS}

Des dépôts de tourbe d'environ 1-1,5 m d'épaisseur se sont accumulés dans des cuvettes mal drainées par-dessus des dépôts marins. Ils sont abondants autour de Kangiqsualujjuaq et plus au sud le long du George de même qu'autour du lac Qarlik. On ne les trouve guère au-delà de la limite actuelle des arbres. Ces tourbières, dont plusieurs renferment des palses (Gahé et al., 1987), ont commencé à s'accumuler il y a environ $5600 \mathrm{BP}$, selon les plus vieilles datations obtenues sur des sédiments organiques basaux.

Les sédiments intertidaux actuels consistent en limons légèrement sableux, à stratifications fines et diffuses et renfermant d'abondants blocs dispersés. Ils s'étalent sur deux niveaux intertidaux, soit un marais en bordure supérieure de l'estran et une vasière à blocs dans la partie inférieure. Ces marais sont le lieu d'une intense activité littorale périglaciaire, incluant l'accroissement du pergélisol (Fournier et al., 1987; Allard et al., 1988).

Les quelques placages éoliens observés ici et là sur des plages soulevées sont peu importants. On retrouvera dans Gahé (1987) une carte à 1/50 000 des formations quaternaires des environs du moyen estuaire et de l'embouchure du George.

\section{LA DÉGLACIATION ET LA LIMITE MARINE}

Après la déglaciation, les régions sur le pourtour de la baie d'Ungava ont été envahies par la Mer d'lberville (Laverdière, 1969). Mesuré en de très nombreux endroits avec un altimètre de précision, le plus haut niveau de cette mer le long de l'estuaire du George se situe à $100 \mathrm{~m}( \pm 1 \mathrm{~m})$ d'altitude. Cette limite marine est facilement identifiable, les terrains au-dessus de ce niveau portant soit des tills épais sous forme de drumlins ou de placages, soit un till d'ablation caillouteux éparpillé sur les affleurements rocheux. La limite inférieure de ce till non remanié, le pied de longs talus de terrasse dans les tills et les plus hautes plages de galets dans les anciennes baies exposées à de grands fetches marquent un peu partout dans la région cette plus haute ligne de rivage. Quelques deltas fluvio-glaciaires ont aussi été édifiés à cette altitude à l'embouchure de rivières qui se déversaient dans la mer (fig. 2 et 7). Ils portent encore sur leur surface les paléo-chenaux divaguants du temps de la déglaciation.

À partir de $185 \mathrm{~m}$, dans la région de Kuujjuaq (Lauriol, 1982), la limite marine s'abaisse donc jusque dans la région étudiée. D'aval en amont, le long du fleuve jusqu'à la chute 

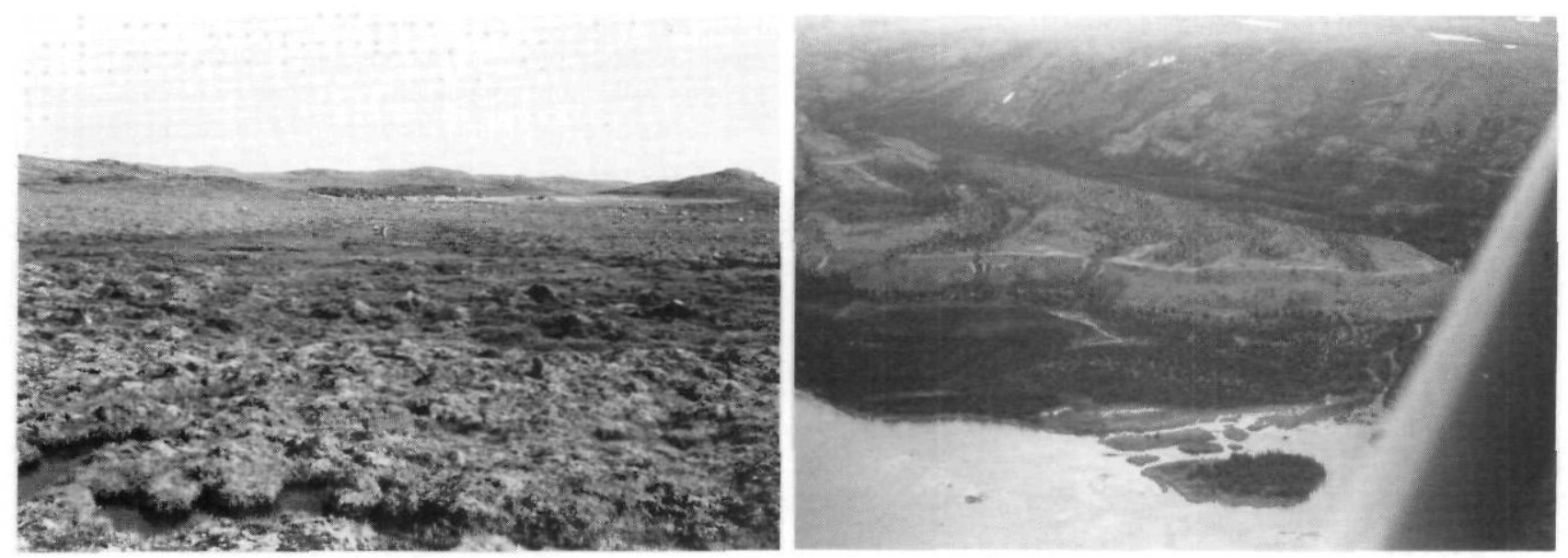

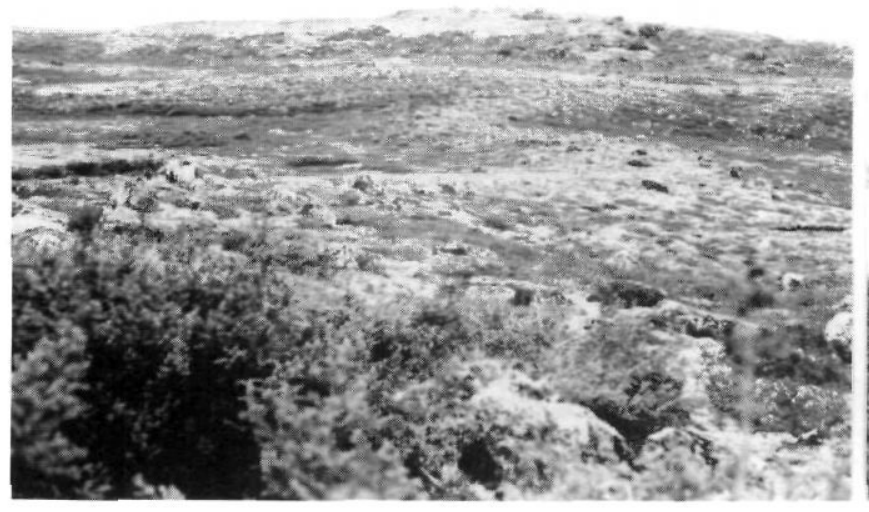

FIGURE 5. Estran soulevé. Le personnage se tient dans un paléochenal de marée.

Raised tidal flat. The person is standing within a raised tidal channel.

FIGURE 6. Crête de blocs glaciels dans la vallée de Kangiqsualujjuaq. À une altitude de $55 \mathrm{~m}$, elle correspond à une importante ligne de rivage holocène.

Boulder barricade across Kangiqsualujjuaq valley. At $55 \mathrm{~m}$ a.s.I., it corresponds to an important Holocene strandline.

Helen, à la confluence de la rivière Ford, le plan de $100 \mathrm{~m}$ est horizontal. À l'embouchure des rivières Barnouin et Koroc, la limite marine n'est plus qu'à $95 \mathrm{~m}$. Elle s'abaisse à $87 \mathrm{~m}$ au lac Qarlik. Au fjord Weymouth, cette limite, clairement visible dans les versants escarpés (fig. 8), est à une altitude de $62 \mathrm{~m}$. On peut en déduire que la déglaciation du cours inférieur du George, sur une distance d'environ 80 km, s'est faite assez rapidement puisqu'il n'y a aucune inclinaison du plan de la limite marine dans le sens nord-sud. Par ailleurs, l'inclinaison du plan vers l'est le long de la côte sud de la baie d'Ungava et vers le nord-est le long de la côte est laisse supposer que les glaciers qui recouvraient les monts Torngat au Wisconsinien supérieur étaient de masse relativement limitée. L'inclinaison du niveau maximal le long de la côte est de la baie d'Ungava suit de façon parallèle celle qui fut relevée par Loken (1962a) le long de la côte atlantique de la péninsule du Labrador.

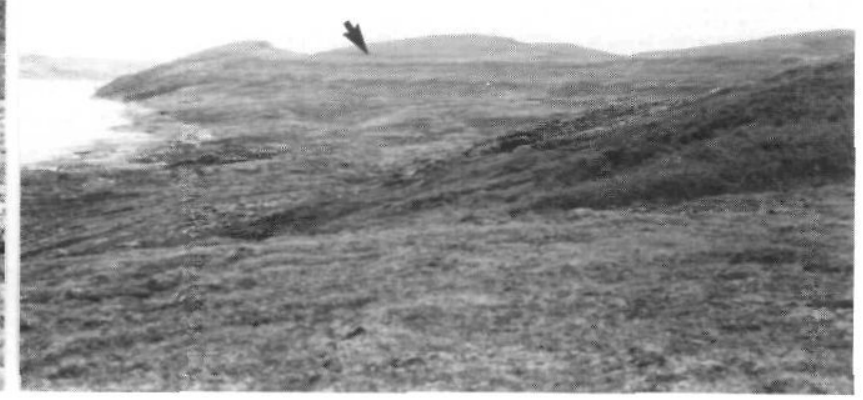

FIGURE 7. Delta fluvio-glaciaire à la limite marine, vallée de la rivière Ford.

Glaciofluvial delta at marine limit in Ford River valley.

FIGURE 8. Limite marine (flèche) et lignes de rivage plus basses le long du fleuve George.

Marine limit and lower shorelines along George River.

Deux datations sur des coquillages marins provenant d'une coupe dans une falaise donnent un âge de 7400-7300 BP (âge au ${ }^{14} \mathrm{C}$ non corrigé) pour la déglaciation du coin sud-est de la baie d'Ungava. Cette coupe est située à la pointe Elson (fig. 2) et comprend, de la base au sommet, trois unités stratigraphiques principales: un till de fond, des limons argileux glacio-marins et des sables et graviers littoraux (fig. 9).

Compte tenu du faciès de fond marin disposé immédiatement sur le till, la date de $7380 \mathrm{BP}$ correspond probablement au tout début de la transgression marine. Cette date est comparable à celles de $7350 \pm 320$ (GY-5093) de Lauriol et al. (1979) et de $7220 \pm 115$ (1-9246) de Gangloff et al. (1976) pour les régions d'Aupaluk et de Quaqtaq (Lauriol et Gray, 1987). L'ensemble des autres dates sur coquilles marines (tabl. I) confirme par ailleurs la contemporanéité de l'invasion marine sur le pourtour de la baie d'Ungava dont les rivages actuels ont donc été déglacés à peu près simultanément. 
TABLEAU ।

Datations au radiocarbone de la région étudiée et des environs immédiats

\begin{tabular}{|c|c|c|c|c|c|c|}
\hline Âge & $\sigma$ & $\mathrm{n}^{\circ}$ laboratoire & & données & altitude $(m)$ & commentaires \\
\hline 270 & 110 & UL-354 & $58^{\circ} 42.7^{\prime} \mathrm{N}$ & $66^{\circ} 02.1^{\prime} \mathrm{W}$ & 2 & Bois flotté \\
\hline 340 & 60 & UL-357 & $58^{\circ} 47.8^{\prime} \mathrm{N}$ & $66^{\circ} 26.7^{\prime} \mathrm{W}$ & 5 & Tourbe basale de thufur \\
\hline 1560 & 50 & UL-353 & $58^{\circ} 42.7^{\prime} \mathrm{N}$ & $66^{\circ} 02.4^{\prime} \mathrm{W}$ & 2 & Bois flotté \\
\hline 1560 & 80 & UL-355 & $58^{\circ} 41.6^{\prime} \mathrm{N}$ & $65^{\circ} 56.1^{\prime} \mathrm{W}$ & 0 & $\begin{array}{l}\text { Bois enfoui dans la vasière au } \\
\text { niveau des hautes mers. } \\
\text { Probablement retransporté }\end{array}$ \\
\hline 2030 & 70 & UL-356 & $58^{\circ} 33.8^{\prime} \mathrm{N}$ & $66^{\circ} 25.9^{\prime} \mathrm{W}$ & 12 & Tourbe basale de thufur \\
\hline 3310 & 210 & UL-267 & $58^{\circ} 53^{\prime} \mathrm{N}$ & $65^{\circ} 46^{\prime} \mathrm{W}$ & 19 & Tourbe basale de palse \\
\hline 3830 & 70 & UL-92 & $58^{\circ} 45.8^{\prime} \mathrm{N}$ & $66^{\circ} 1.3^{\prime} \mathrm{W}$ & 51 & Idem \\
\hline 3940 & 70 & UL-270 & $58^{\circ} 42^{\prime} \mathrm{N}$ & $65^{\circ} 55^{\prime} \mathrm{W}$ & 34 & Idem \\
\hline 4640 & 70 & UL-95 & $58^{\circ} 41.4^{\prime} \mathrm{N}$ & $65^{\circ} 55.3^{\prime} \mathrm{W}$ & 36 & Idem \\
\hline 5120 & 100 & UL-101 & $58^{\circ} 42.9^{\prime} \mathrm{N}$ & $66^{\circ} 0.4^{\prime} \mathrm{W}$ & 34 & Idem \\
\hline 5690 & 150 & UL-98 & $58^{\circ} 34.1^{\prime} \mathrm{N}$ & $65^{\circ} 59.3^{\prime} \mathrm{W}$ & 36 & Idem \\
\hline 5730 & 110 & UL-265 & $58^{\circ} 49.8^{\prime} \mathrm{N}$ & $65^{\circ} 53.5^{\prime} \mathrm{W}$ & 5 & $\begin{array}{l}\text { Coquilles mélangées dans des } \\
\text { limons marins }\end{array}$ \\
\hline 6020 & 230 & UL-263 & $58^{\circ} 42^{\prime} \mathrm{N}$ & $65^{\circ} 58.5^{\prime} \mathrm{W}$ & 24 & $\begin{array}{l}\text { Coquilles de Hiattella arctica } \\
\text { dans sables limoneux } \\
\text { paralittoraux }\end{array}$ \\
\hline 6200 & 200 & QL-1002 & $57^{\circ} 30^{\prime} \mathrm{N}$ & $65^{\circ} 15^{\prime} \mathrm{W}$ & 305 & $\begin{array}{l}\text { Base de sédiments de lac sous } \\
\text { le rivage N4 au pic Pyramide }\end{array}$ \\
\hline 6390 & 90 & UL-264 & $58^{\circ} 52.5^{\prime} \mathrm{N}$ & $65^{\circ} 59^{\prime} \mathrm{W}$ & 21,5 & $\begin{array}{l}\text { Coquilles mélangées au sommet } \\
\text { des argiles à la pointe Elson }\end{array}$ \\
\hline 6720 & 100 & MBN-198 & $58^{\circ} 8^{\prime} \mathrm{N}$ & $67^{\circ} 48^{\prime}$ (approx.) & - & $\begin{array}{l}\text { Coquilles mélangées à la rivière } \\
\text { Aveneau (Gangloff et Pissart, } \\
\text { 1983) }\end{array}$ \\
\hline 6815 & 125 & SI-1959 & $57^{\circ} 38^{\prime}$ & $65^{\circ} 10^{\prime} \mathrm{W}$ & 380 & $\begin{array}{l}\text { Base de sédiments lacustres } \\
\text { près du pic Pyramide (Short, } \\
\text { 1981) }\end{array}$ \\
\hline 7310 & 100 & UL-259 & $58^{\circ} 52.5^{\prime} \mathrm{N}$ & $65^{\circ} 59^{\prime} \mathrm{W}$ & 17 & $\begin{array}{l}\text { Coquilles mélangées à la base } \\
\text { de sédiments marins de la pointe } \\
\text { Elson }\end{array}$ \\
\hline 7380 & 90 & UL-358 & $58^{\circ} 52.5^{\prime} \mathrm{N}$ & $65^{\circ} 59^{\prime} \mathrm{W}$ & 17 & $\begin{array}{l}\text { Ré-échantillonnage pour vérifier } \\
\text { la date UL-259 }\end{array}$ \\
\hline 16800 & 2300 & GX-6387 & $58^{\circ} 28^{\prime} \mathrm{N}$ & $65^{\circ} 10^{\prime} \mathrm{W}$ & 143 & $\begin{array}{l}\text { Base de sédiments lacustres au } \\
\text { lac des Palses; probablement } \\
\text { faussée (Short, 1981) }\end{array}$ \\
\hline
\end{tabular}

\section{LES ÉTAPES DE L'ÉMERSION POSTGLACIAIRE}

En incluant la limite marine, une douzaine de lignes de rivage majeures sont étagées sur les versants des collines et des vallées à travers toute la région. Elles marquent probablement autant de fluctuations eustatiques ou de périodes de ralentissement dans l'émersion des terres. Ces anciens niveaux marins sont à des altitudes de $100 \mathrm{~m}, 96 \mathrm{~m}, 82 \mathrm{~m}$, $62 \mathrm{~m}, 55 \mathrm{~m}, 43 \mathrm{~m}, 35-36 \mathrm{~m}, 32 \mathrm{~m}, 30 \mathrm{~m}, 25-26 \mathrm{~m}, 18 \mathrm{~m}, 12-$ $13 \mathrm{~m}, 9 \mathrm{~m}$ et $4 \mathrm{~m}$ au-dessus du niveau actuel des plus hautes marées (Fournier, 1988). À l'exception de la limite marine qui est particulièrement mise en évidence par le délavage du till sur les versants rocheux, les lignes de rivages holocènes se caractérisent toutes par de nombreuses formes regroupées aux mêmes altitudes: deltas perchés (à $100 \mathrm{~m}$ et à $82 \mathrm{~m}$ ), crêtes de plages de galets et de sables, talus de terrasses et replats, anciens estrans à blocs glaciels, crêtes de blocs glaciels et, même, anciens lambeaux de plates-formes taillées dans le roc (fig. 10) (Fournier, 1988). La figure 11b montre l'étagement de ces formes et leurs correspondances altitudinales le long d'un transect W-E de l'embouchure du George à l'embouchure du Koroc. Alors que tous ces plans sont à 


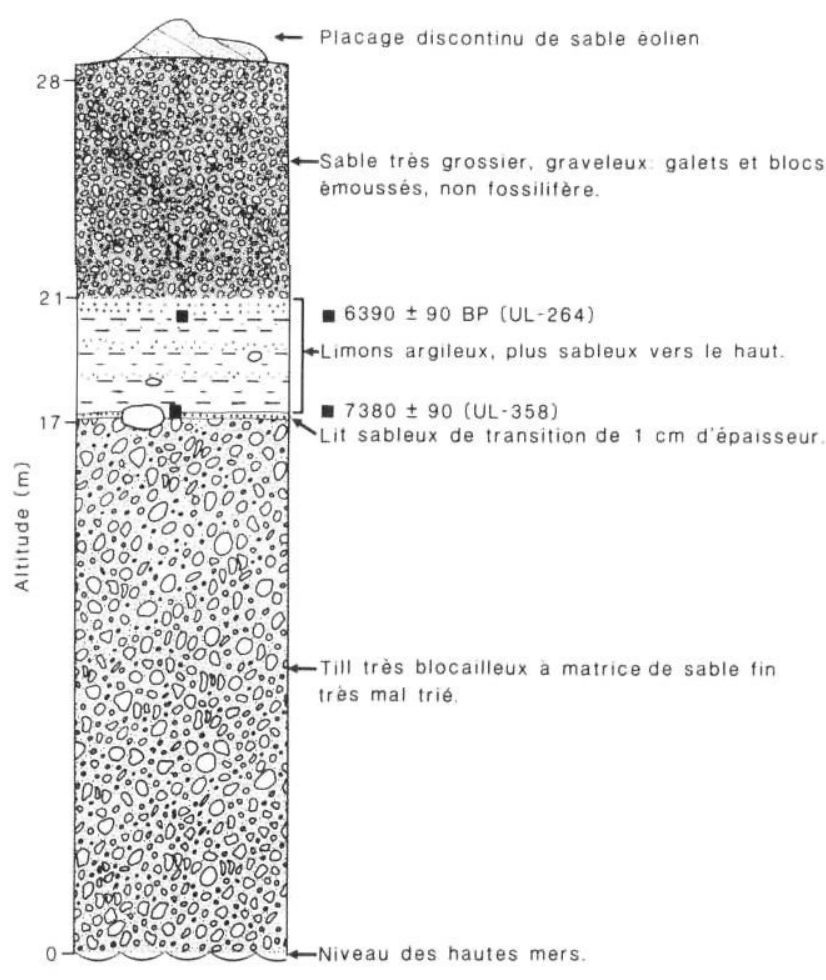

FIGURE 9. Coupe stratigraphique de la pointe Elson.

Stratigraphic section at Point Elson.

la même altitude le long du George, on note que la limite marine et la seconde ligne de rivage sont plus basses à l'embouchure du Koroc. Cependant, les niveaux marins correspondent d'un endroit à l'autre aux altitudes inférieures à $55 \mathrm{~m}$. Si l'on tient pour acquis que la déglaciation est synchrone sur toute la côte, cela implique un relèvement isostatique moindre et plus lent immédiatement après la déglaciation le long de la côte est de la baie, vraisemblablement en raison de la présence de masses de glace moins volumineuses sur la péninsule du Labrador au Wisconsinien supérieur.

La courbe d'émersion proposée (fig. 12) est essentiellement déduite des contraintes logiques imposées par l'interprétation d'une quinzaine de datations sur des coquillages, des tourbes basales et des morceaux de bois de dérive (tabl. I). Un seul échantillon, à $2 \mathrm{~m}$ d'altitude, peut être associé directement à une ligne de rivage. II est approprié d'attribuer à la limite marine les plus vieux âges au ${ }^{14} \mathrm{C}(7380 \pm 90$ et $7310 \pm 100)$ obtenus sur des coquillages même si ceux-ci ne sont qu'à $17 \mathrm{~m}$ d'altitude car, prélevés à la base des sédiments de fond marin, ils représentent la période où, suivant les théories de déglaciation classiques, l'émersion ne faisait que commencer. De plus, cet âge concorde avec l'âge minimal de 6815 $\pm 125 \mathrm{BP}(\mathrm{SI}-1959)$ proposé pour la vidange finale du Lac glaciaire Naskaupi aux collines Pyramides (Ives et al., 1976); en effet, ce lac ne pouvait subsister après la déglaciation de l'estuaire du George et sa disparition devrait correspondre plus ou moins à la fusion, dans la région étudiée, de la glace qui le retenait.

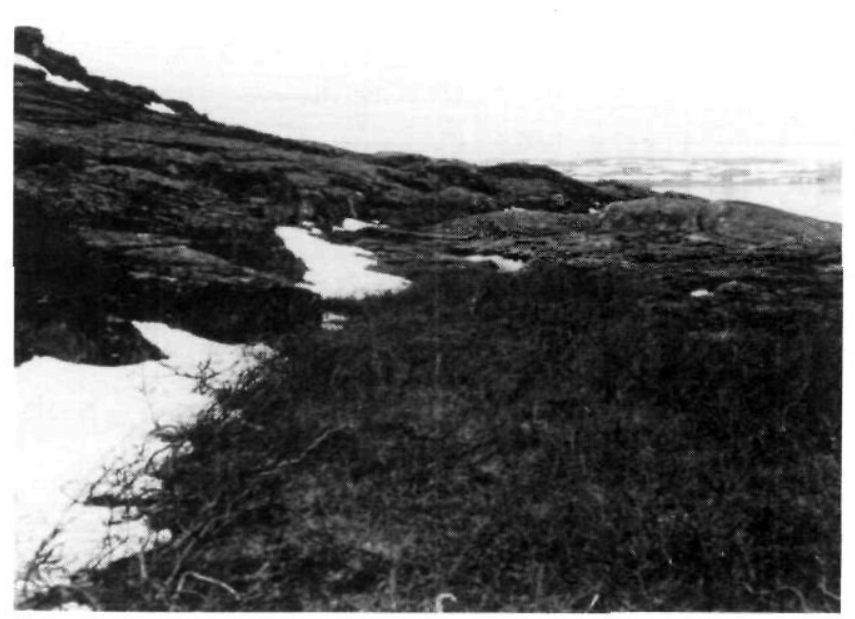

FIGURE 10. Lambeau de plate-forme rocheuse soulevée. La falaise mesure $2 \mathrm{~m}$ de hauteur.

Strech of raised Holocene shoreline platform. The rock bluff is $2 \mathrm{~m}$ high.

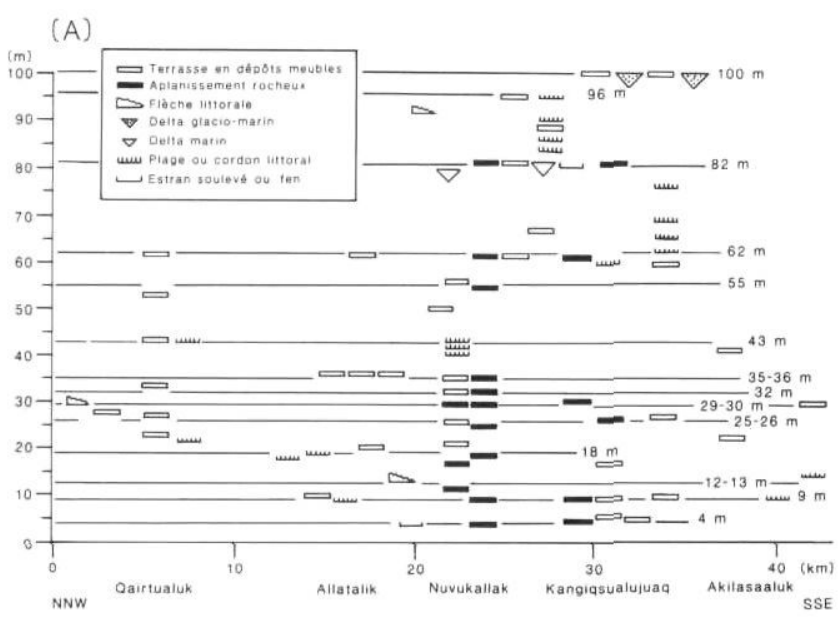

(B)

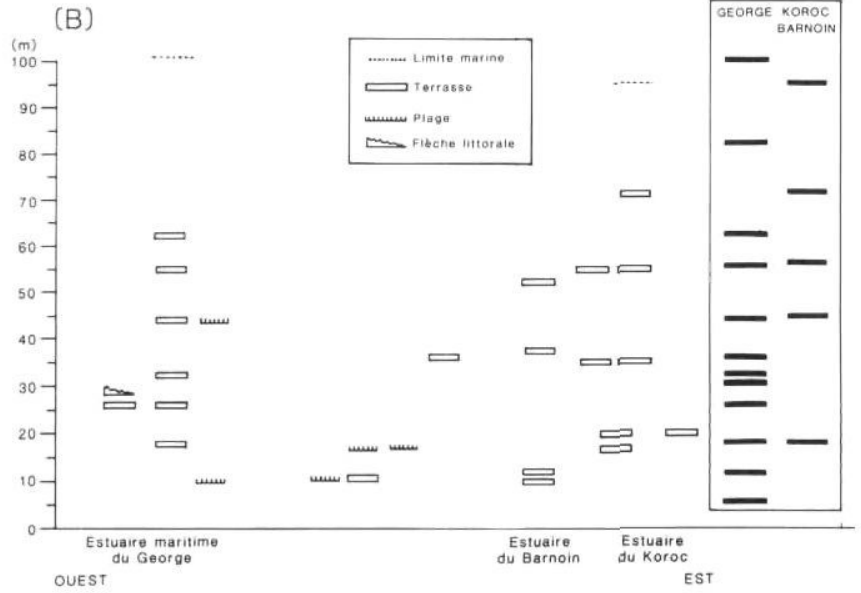

FIGURE 11. Diagrammes de lignes de rivage: a) d'amont en aval le long de l'estuaire du George; b) d'est en ouest entre l'embouchure du George et celle du Koroc.

Shoreline diagrams; a) N-S along George River estuary, b) W-E between George River and Koroc estuaries. 


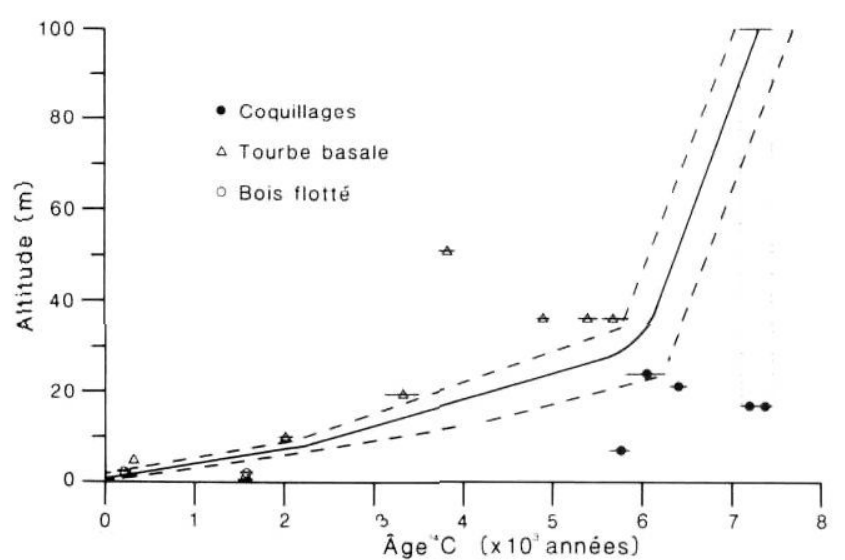

FIGURE 12. Courbe d'émersion de la région de Kangiqsualujjuaq. Sea level curve for the Kangiqsualujjuaq region.

Une inflexion importante de la courbe d'émersion est probable vers 6000-5800 BP selon la logique imposée par deux dates au ${ }^{14} \mathrm{C}$, l'une de $6020 \pm 230$ (UL-263) sur des coquilles marines à $25 \mathrm{~m}$, l'autre de $5690 \pm 150$ (UL-98) sur une tourbe basale à $36 \mathrm{~m}$. L'échantillon de coquilles marines était composé de valves séparées de Hiatella arctica recueillies dans un ravin récemment entaillé le long de la piste d'atterrissage du village de Kangiqsualujjuaq. Les sédiments à cet endroit sont des sables disposés en strates discontinues de granulométries diverses et contenant ici et là des lentilles limoneuses. Ce faciès appartient à un milieu intertidal ou infratidal animé de forts courants de marée tels qu'il en existe encore aujourd'hui et où vit d'ailleurs la même espèce. D'autre part, la tourbe basale implique une émersion préalable. La courbe doit donc passer entre les deux dates et sa marge de fiabilité doit englober les écarts types de la date sur les coquillages qui peuvent avoir vécu en zone intertidale. Ainsi la limite supérieure de fiabilité passe à 5740 BP $($ UL-98 $+1 \sigma)$ et la limite inférieure à $6250 \mathrm{BP}$ (UL-263 + 1\%).

Trois datations sur tourbes basales à des altitudes décroissantes de $19 \mathrm{~m}(3310 \pm 210 \mathrm{BP}, \mathrm{UL}-267)$ à $5 \mathrm{~m}$ (340 \pm 60 BP, UL-357) fournissent des âges minimaux pour l'émersion progressive. La date UL-357 provient de la tourbe basale d'un thufur géant ou turf hummock. Ces cryoformes sont associées aux terrains humides en milieu de pergélisol, constitués principalement par les estrans soulevés (Allard et Seguin, 1987). Le thufur retenu pour fournir un repère chronologique de l'émersion était situé à une centaine de mètres de la ligne actuelle des plus hautes eaux. Ces monticules de 1 à $2 \mathrm{~m}$ de diamètre et d'environ $50 \mathrm{~cm}$ de hauteur ont un cœur minéral injecté vers le haut par la cryoturbation du mollisol et portent une coiffe épaisse de tourbe asséchée et d'éricacées qui, apparemment, continue de s'épaissir suivant le dynamisme végétal après le début de croissance du thufur. Quelques essais de datation de thufurs ont été réalisés dans la région et ont donné des âges jusqu'à 2000 ans sur des sites plus élevés (tabl. I). La quasi totalité des thufurs étudiés montrent un contact franc et non perturbé entre le cœur minéral et la base de la coiffe de tourbe qui, par ailleurs, est la seule source de sédiments organiques basaux que nous avons pu trouver aux très basses altitudes. Malgré quelques craintes sur la validité de cet échantillon, la date obtenue à $5 \mathrm{~m}$ concorde bien avec celle obtenue sur du bois de dérive à $2 \mathrm{~m}$ d'altitude .

Cet échantillon de bois, âgé de $270 \pm 110$ (UL-354), consiste en quelques petits fragments trouvés dans une fissure du roc comblée par des galets émoussés à une vingtaine de mètres de distance de la crête de tempête actuelle. Associés au bois se trouvaient quelques restes quétineux de vers marins, appartenant probablement au genre Tubularia (D. Calder, Royal Ontario Museum, comm. écrite, 1987); il s'agit d'organismes qui se fixent au bois de dérive, aux bouées et aux structures immergées ( $E$. Bourget, Département de biologie, Université Laval, comm. pers., 1987). Leur présence indique qu'il s'agit bien de bois de dérive. Le faible diamètre $(8 \mathrm{~mm})$ des morceaux de bois assure que l'âge à leur mort ne dépasse pas la marge d'erreur de la datation qui, par conséquent, peut être jugée acceptable pour ce niveau marin. Par ailleurs, deux datations de 1560 BP (tabl. I et fig. 12) ont été obtenues sur du bois de dérive à $2 \mathrm{~m}$ et enfoui dans la vase au niveau des hautes marées. Bien que l'on puisse envisager la possibilité qu'il s'agisse de bois ancien redéposé, il demeure possible que le taux d'émersion ait été encore plus lent que calculé depuis 1600 ans, voire même que quelques fluctuations se soient produites récemment.

La courbe obtenue, avec ses marges de précision estimées, montre une émersion relativement rapide à partir de la déglaciation jusqu'à environ 6000-5800 BP (fig. 12). Le taux d'émersion, initialement de l'ordre de $4,5 \mathrm{~m} /$ siècle passe à $0,5 \mathrm{~m} /$ siècle après la période charnière. Malgré l'absence de datations de spécimens marins entre 5000 et $2000 \mathrm{BP}$, l'émersion semble linéaire depuis.

L'espacement altitudinal entre les lignes de rivage soulevées (fig. 11) devient faible aux altitudes inférieures à $40 \mathrm{~m}$ et, plus précisément, après la formation de l'important rivage de $36 \mathrm{~m}$. Ce changement du taux d'émersion en est la cause. De plus, il devient difficile aux basses altitudes de préciser sur le terrain si certaines formes altitudinalement proches les unes des autres appartiennent à un seul ou à deux niveaux marins anciens, compte tenu du marnage marégraphique de l'ordre de 8 à $13 \mathrm{~m}$ qui prévaut dans la région.

Soulignons finalement que la période charnière observée dans la courbe d'émersion est synchrone sur plusieurs courbes du Québec nordique (Deception, Aupaluk, Quaqtaq; Lauriol, 1982; Mathews, 1967; Gray et al., 1980) ce qui laisse supposer une influence à l'échelle régionale sur le relèvement isostatique. Ce ralentissement subit de l'émersion vers 6000-5800 BP pourrait être associé à la disparition très rapide des masses de glace qui surchargeaient la croûte terrestre dans le centre du Québec-Labrador. Cependant, les dates sur sédiments lacustres les plus proches de la déglaciation dans les environs de Schefferville sont de $6685 \pm 205$ (GX-6975) et $6345 \pm 160$ (Beta-1201) (Stravers, 1981); il existerait donc un décalage d'environ 600-700 ans entre la déglaciation finale et le point d'inflexion des courbes d'émersion. Ce déphasage pourrait correspondre à la période de soulèvement très rapide du centre de la péninsule lors de sa déglaciation. Le ralentissement subséquent se serait ensuite étendu à la périphérie, le long des côtes. 


\section{DISCUSSION}

Les nouvelles observations recueillies dans la région au sud-est de la baie d'Ungava aident à élucider certaines données radiochronologiques contreversées existantes. Elles ne correspondent pas, non plus, aux concepts de déglaciation couramment énoncés.

\section{CHRONOLOGIE}

Une datation au ${ }^{14} \mathrm{C}$ de $16800 \pm 2300$ (GX-6387) a été obtenue sur un échantillon recueilli par Nichols (in Short, 1981), à la base des sédiments du lac des Palses (nom non officiel) dans le bassin de la rivière Barnouin à environ $22 \mathrm{~km}$ à l'est de la région couverte par nos relevés. Le lac échantillonné n'est qu'à 143 m d'altitude. S'il fallait retenir cette date pour la déglaciation locale, il est évident que la région étudiée, dont l'altitude moyenne est supérieure, aurait été déglacée bien avant $8000 \mathrm{BP}$. Ce lac se situe à peine à $45 \mathrm{~km}$ à vol d'oiseau de la pointe Elson où des coquillages marins ont été datés entre 7400 et $6400 \mathrm{BP}$. Ces derniers âges concordent avec la chronologie ungavienne (Lauriol et Gray, 1987) et proviennent d'un matériau datable abondant et fiable.

Dans l'état actuel des connaissances, nous devons présumer que la datation de ces sédiments lacustres, comme d'autres dans la région de la rivière Swampy Bay (Short, 1981) est faussée. Ces datations anormalement vieilles résultent peut-être d'une contamination des sédiments lacustres minéraux, à la base de la gyttja, par un peu de matière organique datant du Wisconsinien moyen ou inférieur (Stravers, 1981). Une telle contamination, minime en quantité absolue, devient en effet très importante dans le cas de ces échantillons qui ne contiennent qu'une infime teneur en matière organique $(<1 \%)$.

\section{CONCEPTS DE LA DÉGLACIATION}

Les orientations des stries et des drumlins entre le fjord Weymouth et le fleuve George indiquent que le dernier écoulement glaciaire provenaient du sud-est, soit des monts Torngat. Cet écoulement s'associe au «Ancestral Labrador Ice Divide", de Dyke et Prest (1987) c'est-à-dire une crête dans l'inlandsis localisée sur ces hauts sommets et s'écoulant d'une part jusqu'à l'Atlantique via les fjords tout en laissant des sommets non englacés (Evans et Rogerson, 1986; Josenhans et al., 1986) et, d'autre part, vers le bassin de la baie d'Ungava. Dyke et Prest (1987) croient que cette ligne de partage a existé du maximum du Wisconsinien supérieur jusque vers 9000 BP.

Selon le modèle proposé par Ives, 1960c (voir aussi Ives et al., 1976; Ives et al., 1975) et repris sur les cartes paléogéographiques de Dyke et Prest (1987), cet écoulement glaciaire régional aurait été supplanté à l'approche de la déglaciation par une masse de glace couvrant la baie d'Ungava et dont la direction d'écoulement dans la région étudiée aurait dû être vers l'est. Cette dernière masse glaciaire sur la baie et le cours inférieur du George est invoquée pour retenir le Lac glaciaire Naskaupi dans la vallée supérieure du George et le Lac glaciaire McLean dans celle de la rivière à la Baleine (voir aussi Barnett, 1963, 1967). Or, comme le mentionnent
Dyke et Prest (1987): The eastward flow to the ice margin holding Lake Naskaupi is problematic because what appears to be the last ice flow direction in that area... is from the lake basin towards the northwest. Either a later eastward flow was incapable of remolding the drift surface or the pattern of retreat is different than figured. " (p. 253). Matthew (1961a) avait vu le problème autour des collines Pyramides, secteur où le lac devait être retenu à son extrémité nord.

Selon les informations fragmentaires disponibles, l'importante masse de glace canalisée par le détroit d'Hudson traversait l'entrée de la baie d'Ungava, s'écoulait vers l'est et débordait sur la marge de l'Atlantique à travers la péninsule du Labrador avant 9000 BP, date minimale de la déglaciation à Eclipse Channel (Loken, 1962a et b). Puis elle a reculé, abandonnant les moraines de Sheppard au centre de la péninsule, au sud de Killiniq. En direction tout à fait opposée, les derniers écoulements glaciaires entre le fjord Abloviak et le George se sont dirigés vers la baie d'Ungava et en ont épousé la configuration. Tout en s'amincissant, le glacier dans le coin sud-est de la baie d'Ungava devait donc reculer vers le sud-est en direction du Koroc central (Savoie et al., 1980; Pilon et al., 1980). Néanmoins, on doit aussi envisager le recul d'un front glaciaire vers la baie d'Ungava pour expliquer la rétention de lacs glaciaires dans la vallée du Koroc et du Barnouin (Barré, 1984). La seule hypothèse à retenir pour l'instant pour concilier ses données contradictoires est que la dernière masse glaciaire à avoir occupé le cours inférieur du George n'était animée d'aucun écoulement lors de la fonte finale. Les directions représentées aujourd'hui par les stries et les drumlins doivent donc dater d'avant la fonte finale.

Par ailleurs, les datations sur coquillages indiquent une déglaciation synchrone sur tout le pourtour de la baie d'Ungava, plutôt qu'un décalage entre les rives est et ouest comme le veut I'hypothèse habituelle. Le tracé général des écoulements glaciaires autour de la baie (Lauriol, 1982; Gangloff et al., 1976; Bouchard et Marcotte, 1986; Drummond, 1965) laisse croire, ainsi que la présente étude, que la déglaciation rapide de la baie d'Ungava a dû, au contraire, créer un appel centripète du flux glaciaire vers la Mer d'lberville qui s'est ouverte très rapidement. C'est alors que des réajustements dans les masses glaciaires se sont produits et qu'ont été provoqués les derniers changements locaux de direction d'écoulement glaciaire, comme le déplacement angulaire de $20^{\circ}$ observé dans la région et les réorientations finales observées au sud de Kuujjuaq (Gangloff et Pissart, 1983, Gangloff et al., 1976, Drummond, 1965, p. 104).

\section{CONCLUSION}

Une explication satisfaisante de l'évolution des lacs glaciaires du bassin versant supérieur de la baie d'Ungava, en particulier ceux du Kaniapiscau (lac Cambrien; Drummond 1965), de la Baleine (Lac McLean), du George (Lac Naskaupi) de même que ceux du Koroc (Barré, 1984), exige que l'on fasse encore de nombreux relevés de terrain sur le vaste territoire encore non étudié. La chronologie relative et absolue de la déglaciation du détroit d'Hudson, de la baie d'Ungava et du nord-est du Québec-Labrador reste à établir afin que 
l'on puisse réconcilier dans le temps et dans l'espace les directions d'écoulement glaciaire et les plans glacio-lacustres (Matthew, 1961b). Une partie importante de l'information requise devra peut-être provenir de l'étude du fond de la baie d'Ungava. De plus, l'étude de la composition lithologique du till serait susceptible de fournir plus de renseignements sur les mouvements glaciaires antérieurs. Les données de la présente étude ne sont encore qu'une pièce du casse-tête.

\section{REMERCIEMENTS}

Les auteurs remercient sincèrement les nombreux étudiants et auxiliaires qui ont participé aux séjours sur le terrain depuis 1984: $M^{\text {mes }}$ Jacqueline Bouchard, Florence Nicollin, Lyne Messier, Renée Plourde, Johanne Plourde et MM. Yvon Pelletier, Jean Desbiens, Richard Fortier, Fernando Sheriff, Richard Lévesque et Christian Bouchard. $\mathrm{M}^{\text {me }}$ Andrée Lecours et $\mathrm{M}$. Richard Turcotte ont procédé aux datations au ${ }^{14} \mathrm{C}$. Le professeur Edwin Bourget du Département de biologie de I'Université Laval et le $D^{r}$ Dale R. Calder du Musée royal de l'Ontario ont gracieusement identifié les restes de vers marins fossiles. Les auteurs ont bénéficié de discussions sur le terrain avec M. R. A. Klassen de la Commission géologique du Canada et le regretté collègue Guy Lortie. Les illustrations ont été dessinées par MM. Yvan P. Grenier et Stephen Poitras. Des améliorations ont été apportées au texte à la suite des commentaires de MM. Jean-Serge Vincent et Pierre Gangloff, les deux lecteurs de la revue. Cette étude fait partie d'un programme de recherche à long terme que nous avons établi dans la région et qui est appuyé financièrement par le Fonds FCAR du Québec, le CRSNG, le programme des conventions de recherche de la Commission géologique du Canada et le programme de formation scientifique du ministère des Affaires indiennes et du Nord canadien. Les auteurs tiennent à souligner la qualité de l'accueil et l'esprit d'amitié dispensés par les gens de Kangiqsualujjuaq.

\section{RÉFÉRENCES}

Allard, M., Seguin, M. K. et Pelletier, Y., 1988. Shoreline permafrost in Kangiqsualujjuaq Bay Ungava, Québec. Proceedings, Fifth International conference on Permafrost, Trondheim, Norvège, p. 113118.

Allard, M. et Seguin, M. K., 1987. Le pergélisol au Québec nordique: bilan et perspectives. Géographie physique et Quaternaire, 41 : 141-152.

Barnett, D. M., 1963. Former pro-glacial lake shorelines as indicators of the pattern of deglaciation of the Labrador-Ungava Peninsula. McGill Sub-Arctic Research Papers, 15: 23-33.

1967. Glacial Lake McLean and its relationships with glacial lake Naskaupi, Geographical Bulletin, 9 (2): 96-101.

Barré, D., 1984. Cartographie géomorphologique détaillée appliquée à la région đu mont Nuvulialuk et đu Koroc aval, Nouveau-Québec. Mémoire M.Sc. Université de Montréal, Département de géographie, $173 \mathrm{p}$.

Bouchard, M. A. et Marcotte, C., 1986. Regional glacial dispersal patterns in Ungava, Nouveau-Québec. In Current Research, Part B, Geological Survey of Canada, Paper 86-1 B, p. 295-304.
Drummond, R. M., 1965. The glacial geomorphology of the Cambrian Lake area, Labrador-Ungava. Ph.D. Thesis, McGill University, Montréal, $222 \mathrm{p}$.

Dyke, A. S. et Prest, V. K., 1987. Late Wisconsinan and Holocene history of the Laurentide Ice Sheet. Géographie physique et Quaternaire, $41:$ 237-264.

Evans, D. J. A. and Rogerson, R. J., 1986. Glacial geomorphology and chronology in the Selamint Range-Nachvak Fiord area, Torngat Mountains, Labrador. Canadian Journal of Earth Sciences, 23: 66-76.

Fournier, A., 1988. Les processus d'érosion périglaciaire des estrans rocheux de l'estuaire médian du George, Québec nordique. Département de géographie, Université Laval, thèse de maîtrise, $194 \mathrm{p}$.

Fournier, A., Allard, M. et Seguin, M. K., 1987. Typologie morphogénétique des marelles du marais littoral de la baie de Kangiqsualujjuaq, estuaire du George, Québec nordique. Géographie physique et Quaternaire, 41: 47-64.

Gahé, É., 1987. Géomorphologie cryogène et géophysique dans la région de Kangiqsualujjuaq, Ungava. Thèse de Ph.D. Université Laval, Département de géographie, $210 \mathrm{p}$.

Gahé, É., Allard, M. et Seguin, M. K., 1987. Géophysique et dynamique holocène de plateaux palsiques à Kangiqsualujjuaq, Québec nordique. Géographie physique et Quaternaire, 41: 33-46.

Gangloff, P., Gray, J. T. et Hillaire-Marcel, C., 1976. Reconnaissance géomorphologique sur la côte ouest de la baie d'Ungava. Revue de géographie de Montréal, 30: 339-348.

Gangloff, P. et Pissart, A., 1983. Évolution géomorphologique et palses minérales près de Kuujjuaq (Fort-Chimo, Québec). Bulletin de la Société géographique de Liège, 19: 119-132.

Gray, J., de Boutray, B., Hillaire-Marcel, C. et Lauriol, B., 1980. Postglacial emergence of the west coast of Ungava Bay, Québec. Arctic and Alpine Research, 12: 19-30.

Henderson, E. P., 1959. A glacial study of central Québec-Labrador. Geological Survey of Canada, Bulletin, 50, $94 p$.

Ives, J. D., 1960a. Former ice-dammed lakes and the deglaciation of the middle reaches of the George River, Labrador-Ungava. Geographical Bulletin, 14: 44-70.

_ 1960b. Glaciation and deglaciation of the Helluva Lake area, Central Labrador-Ungava. Geographical Bulletin, 15: 46-64.

_ 1960c. The deglaciation of Labrador-Ungava: an outline. Cahiers de géographie de Québec, 4: 323-343.

_ 1978. The maximum extent of the Laurentide ice sheet along the east coast of North America during the last glaciation. Arctic, 31: 24-53.

Ives, J. D., Andrews, J. T. et Barry, R. G., 1975. Growth and decay of the Laurentide ice sheet and comparisons with FennoScandinavia. Die Naturwissenschaften, 62: 118-125.

Ives, J. D., Nichols, H. and Short, S. K., 1976. Glacial history and paleoecology of northeastern Nouveau-Québec and Northern Labrador. Arctic, 29: 48-52.

Josenhans, H., Zevenhuigen, S. and Klassen, R. A., 1986. The Quaternary geology of the Labrador Shelf. Canadian Journal of Earth Sciences, 23: 1190-1213.

Lauriol, B., 1982. Géomorphologie et Quaternaire du sud de l'Ungava. Collection Paléo-Québec, n 15, $173 \mathrm{p}$.

Lauriol, B., Gray, J. T., Hétu, B. et Cyr, A., 1979. Le cadre chronologique et paléogéographique de l'évolution marine depuis la déglaciation 
dans la région d'Aupaluk, Nouveau-Québec. Géographie physique et Quaternaire, 33: 189-203.

Lauriol, B. et Gray, J. T., 1987. The decay and disappearance of the late Wisconsin ice sheet in the Ungava peninsula, Northern Québec, Canada. Arctic and Alpine Research, 19: 109-126.

Laverdière, C., 1969. Mer d'lberville. Revue de Géographie de Montréal, 23: 358.

Loken, O., 1962a. The late glacial and post-glacial emergence and the deglaciation of northernmost Labrador. Geographical Bulletin, 17: 23-56.

$1962 b$. On the vertical of glaciation in northeastern LabradorUngava. Canadian Geographer, 6: 106-119.

1964. A study of the late and postglacial changes of sea level in northern most Labrador. Typed report to the Arctic Institute of North America, $80 \mathrm{p}$.

Matthew, E. M., 1961a. The glacial geomorphology and deglacierization of the George River basin. McGill University, Sub-Arctic Research Laboratory, Annual Report 1959-60, Research Paper, 11: 29-45.

1961b. Deglaciation of the George River Basin, LabradorUngava. In J. T. Andrews and E. M. Matthew, Geomorphological studies in northeastern Labrador-Ungava. Department of Mines and Technical Surveys of Canada, Geographical Branch, Geographical Paper, 29: 17-29.

Mathews, B., 1967. Late Quaternary land emergence in northern Ungava. Arctic, 10: 176-202.

Pilon, D., Savoie, L. et Gangloff, P., 1980. Géomorphologie quaternaire de la région du Grenier, Québec-Labrador. Annales de l'ACFAS, 47 (1).

Prest, V. K., Grant, D. R. et Rampton, V. N., 1968. Glacial map of Canada. Geological Survey of Canada, map 1253 A.
Québec, 1983. Les parcs québécois. 7. Les régions naturelles. Gouvernement du Québec, Ministère du Loisir, de la Chasse et de la Pêche, $257 \mathrm{p}$.

Richard, P. J. H., Larouche, A. et Bouchard, M. A., 1982. Âge de la déglaciation finale et histoire postglaciaire de la végétation dans la partie centrale du Nouveau-Québec. Géographie physique et Quaternaire, 36: 63-90.

Savoie, L., Pilon, D. et Gangloff, P., 1980. Notes sur la déglaciation de la péninsule du Québec-Labrador, Annales de l'ACFAS, 47 (1).

Short, S. K., 1981. Radiocarbon list I. Labrador and Northern Québec, Canada. University of Colorado, Institute of Arctic and Alpine Research, Occasional Paper $n^{\circ} 36,33 p$.

Stravers, L. K. S., 1981. Palynology and deglaciation history of the Central Labrador-Ungava. M.Sc. thesis University of Colorado, Institute of Arctic and Alpine Research, Department of Geological Sciences, $171 \mathrm{p}$.

Taylor, F. C., 1971. A revision of Precambrian structural provinces in northeastern Québec and northern Labrador. Canadian Journal of Earth Sciences, 8: 579-584.

1974. Reconnaissance geology of a part of the Precambrian Shield, northeastern Québec, northern Labrador and Northwest Territories. Geological Survey of Canada, Paper 74-21, 9 p.

Veillette, J. J., 1986. Former southwesterly ice flows in the AbitibiTémiskaming region: implications for the configuration of the late Wisconsinan in sheet. Canadian Journal of Earth Sciences, 23 : 1724-1741.

Veillette, J. J. et Nixon, F. M., 1980. Portable drilling equipment for shallow permafrost sampling. Geological Survey of Canada, Paper 79-21, $35 \mathrm{p}$. 